\title{
Active School Travel is Associated With Fewer Suicide Attempts Among 127, 097 Adolescents Aged 13 to 17 Years From 34 Low-and Middle- income Countries
}

\author{
Si-Tong Chen \\ Victoria University \\ Qian Yu \\ Shenzhen University \\ Brendon Stubbs
}

Texas A\&M University College Station

Cain Clark

Coventry University

\section{Zhihao Zhang}

Shenzhen University https://orcid.org/0000-0002-3634-453X

\section{Mingyue Zhu}

Shenzhen University

\section{Md Mahhub Hossain}

Texas A\&M University College Station

\section{Albert Yeung}

Massachusetts General Hospital

\section{Mark D. Griffiths}

Nottingham Trent University

Liye Zou ( $\sim$ liyezou123@gmail.com )

School of Psychology, Shenzhen University

\section{Research}

Keywords: active travel, mental health, suicidality, low and middle income, theoretical study

Posted Date: August 26th, 2020

DOI: https://doi.org/10.21203/rs.3.rs-64099/v1

License: (c) (1) This work is licensed under a Creative Commons Attribution 4.0 International License. Read Full License 
Page $2 / 18$ 


\section{Abstract}

Background. This study explored the association between active school travel (AST) and suicide attempts among adolescents in low- and middle-income countries.

Methods. We used the data from the Global School-based Health Survey, including 127, 097 adolescents aged 13-17 years from 34 LMICs. A self-reported survey was used to collect data on AST and suicide attempts as well as some key control variables. Multivariable logistic regression was performed to assess the association between AST and suicide attempts. A meta-analysis with random effects was undertaken to identify the difference in the association between AST and suicide attempts.

Results. Across all the adolescents, the prevalence of AST was $37.0 \%$ and the prevalence of suicide attempts was $11.6 \%$. Adolescents who engaged in AST were less likely to have suicide attempts irrespective of gender. The country-wise analysis indicated a large inconsistency in the association between AST and suicide attempt across the countries.

Conclusions. AST would appear to be a protective factor for reducing suicide attempts among adolescents in LMICs. However, the association between AST and suicide attempts varied greatly across the countries. Future studies should confirm or negate the association between AST and suicide attempts.

\section{Background}

Suicide among school-aged children and adolescents represents the second biggest contributor to global mortality, with more than 200,000 deaths recorded [1]. Approximately $80 \%$ of suicidal cases among adolescents occur in low- and middle-income countries (LMICs) [1]. Notably, a history of previous suicide attempts represents one of the strongest predictors of further suicide attempts and completed suicide, and this association has been previously reported in some studies that included young populations [2, 3]. Therefore, identifying potentially modifiable risk factors (e.g., behavioral, social, and psychological) to reduce suicide attempts among adolescents is a public health priority, particularly in LMICs [4-6].

A large body of data, collated over previous decades, has demonstrated well-established associations between lifestyle factors (e.g., physical activity [PA], sedentary behavior, and sexual behavior) and suicide attempts $[4,5,7,8]$. Some studies have focused on the role of PA in preventing suicide attempts, with one meta-analysis indicating that active participation in PA can be a protective factor against suicide attempts among adolescents [9]. For example, a US study with an adolescent sample $(n=13,583)$ found that students who reported exercising 4 to 5 days per week had significantly lower odds of suicide attempts than those who exercised 0 to 1 day per week [10]. In addition, Felez-Nobrega et al. [7] recently reported that a higher level of habitual PA was less likely to be associated with suicide attempts (odds ratio $=0.78,95 \% \mathrm{Cl}: 0.70-0.86$ ) in boys, but not girls. Conversely, some studies have indicated that PA does not have any protective effect on suicide attempts among adolescents. For example, a large-scale US study ( $n=65,182$ middle-school students) found no association between overall PA levels and suicide 
attempts [11]. Chinese researchers also reported similar findings and concluded that increased PA might not be a protective factor against suicide attempts [12]. Counter-intuitively, one study reported that frequent high-intensity PA might be associated with an increased odds for suicide attempts among Korean adolescents [13]. These differing results might due to the type of PA engaged or respective contexts. Against this background, It is urgently needed explore the clear associations between some specific types of PA and suicide attempts among adolescents. Explorations of this research issue would be valued because the evidence can provide more practical implications and effective strategies for suicide preventions. Furthermore, considering the complexity of PA, identifying its components with positive roles in reducing suicide attempts is critically valuable.

Active school travel (AST; i.e., walking or riding bicycles between home and school), is an important component of PA that commonly occurred in daily life, particularly among adolescents $[14,15]$. It is wellknown that AST is associated with numerous physical health benefits among youths such as improvements in, or maintenance of, body composition and cardiorespiratory fitness $[16,17]$. Recently, both Eastern and Western researchers have started to investigate the association of AST with mental health outcome, suggesting that children and adolescents engaging in AST have lower odds of developing mental problems like depression $[18,19]$. Because of the potential psychological benefits resultant from AST [20], especially in reducing depression which is one of the key predictors of suicide attempts [21], it is conceivable that AST could be an effective approach in regulating such predictors (e.g., depression, anxiety, and loneliness) that are closely associated with greater odds of suicide attempts among children and adolescents [22-25], leading to a reduced probability of committing suicide. However, little research has directly established association between AST and suicide attempts among schoolbased children and adolescents who experience multifaceted pressures including academic stress and problems in interpersonal relationships. Furthermore, there is an absence of multi-country and LMIC research investigating associations between AST and suicide attempts. Owing to the rising number of suicides in LMICs in recent years [26], more feasible ways to prevent adolescent suicide attempts is needed.

Indeed, if the veracity of the association between AST and suicide attempts can be demonstrated, more effective and efficient interventions, for preventing suicide could be implemented. Therefore, given the dearth of research, the present study investigated the relationship between AST and suicide attempts by using data from 34 LMICs from the World Health Organization (WHO) regions.

\section{Methods}

\subsection{The survey}

Publicly available data from the Global School-based Student Health Survey (GSHS) were analyzed in this study. Details on this survey can be found at http://www.who.int/chp/gshs and http://www.cdc.gov/gshs, respectively. Briefly, the GSHS was jointly developed by the World Health Organization and the United States Centers for Disease Control and Prevention and other United Nations 
allies. The core aim of the survey was to assess and quantify risk and protective factors of major noncommunicable diseases. The survey used a standardized two-stage probability sampling design for the selection process within each participating country. For the first stage, schools were selected with probability proportional to size sampling. The second stage involved the random selection of classrooms that included students aged $13-15$ years, within each selected school. All students in the selected classrooms were eligible to participate in the survey, and data collection was performed during one regular class period. The questionnaire was translated into the local language in each country and consisted of multiple-choice response options. Students recorded their responses on computer scannable sheets. All GSHS surveys were approved, in each country, by both a national government administration (most often the Ministry of Health or Education) and an institutional review board or ethics committee. Student privacy was protected through anonymous and voluntary participation, and informed consent was obtained, as appropriate, from the students, parents, and/or school officials. Data were weighted for non-response and probability selection. From all the publicly available data, we selected all datasets that included the variables pertinent to our analysis. If there were more than two datasets from the same country during this period, we chose the most recent dataset. A total of 34 countries were included in the current study, and, based on the World Bank classification at the time of the survey, all countries were classed as LMICs. Data were nationally representative for all countries, with the exception of some countries. The characteristics of each country, including the sample size and the response rate are provided in Table 1.

\subsection{Active school travel (exposure)}

Active school travel was assessed by a single question: "How many days did you walk or ride a bicycle to and from school during the past 7 days?". Participants self-reported their responses according to their actual situations (responses: $0-7$ days, as integers). Participants having 5 days or more for walking or cycling were regarded as being active, whereas those not reporting 5 days or more for walking or cycling were regarded as being passive. The rationale for selecting 5 days or more, is that adolescents across different countries normally have a minimum of 5 days for schooling per week, which is in line with our previous (as yet unpublished) studies.

\subsection{Suicide attempt (outcome)}

Suicide attempts were assessed by the question "During the past 12 months, how many times did you actually attempt suicide ?", and was defined as at least one suicide attempt in the past 12 months, which is in line with previous research [27].

\subsection{Control variables}

Gender, age, physical activity, sedentary behavior, presence of close friends, anxiety-induced insomnia, fruit and vegetable intake, fast food consumption, and alcohol consumption, as well as food insecurity, were used as control variables in the analysis, based on previous studies. To assess levels of physical activity, questions that represented the PACE+ Adolescent Physical Activity Measure were asked, which 
has been shown to be reliable and valid Questions were asked to ascertain the number of days where at least 60 minutes of physical activity during the past 7 days, not including physical activity during physical education or gym classes, was achieved [28]. Sedentary behavior was assessed with the question "How much time do you spend during a typical or usual day sitting and watching television, playing computer games, talking with friends, or doing other sitting activities?" with answer options: $<1$, $1-2,3-4,5-6,7-8$, or $\geq 8 \mathrm{~h} /$ day. The presence of close friends was defined as having at least one close friend. Anxiety-induced insomnia was assessed with the question: "During the past 12 months, how often have you been so worried about something that you could not sleep at night?" with answer options: 'never', 'rarely', 'sometimes', 'most of the time', and 'always'. Those who answered 'most of the time' or 'always' were considered to have anxiety-induced insomnia.

As in previous research using the GSHS datasets [27, 29], food insecurity was treated as a proxy for socioeconomic status as no variables on socioeconomic status were included in the GSHS datasets. Food insecurity was assessed by the question "During the past 30 days, how often did you go hungry because there was not enough food in your home?" Answer options were in five categories: "never", "rarely", "sometimes", "most of the time" and "always".

\subsection{Statistical analyses}

All statistical analyses were performed using R ( $R$ Core Team, version 3.6.1, 2019). Descriptive analysis was used to calculate the prevalence of AST (defined as occurring at least 5 days a week) and suicide attempt (defined as at least once over the past 12 months). The prevalence of AST and suicide attempts by country were also calculated. Multivariable logistic regression analysis was utilized to assess the association between AST and suicide attempts (overall, by gender and country). The variables of AST and suicide attempt were treated as binary. The regression analysis was adjusted for gender, age, food insecurity, presence of close friends, anxiety-induced insomnia, fruit and vegetable intake, fast food consumption, alcohol consumption, physical activity and sedentary behavior, as well as country, with the exception of the gender-stratified and country-wise analyses which were not adjusted for gender and country, respectively. To assess the level of between-country heterogeneity, the Higgin's $P$ statistic was calculated based on country-wise estimates. This represents the degree of heterogeneity that is not explained by sampling error with a value of $<40 \%$ often considered as negligible, and $40-60 \%$ as moderate heterogeneity [30]. A pooled estimate was obtained by combining the estimates for each country into a random effect meta-analysis. All variables were included in the regression analyses as categorical variables, with the exception of age and PA which were treated as continuous variables. Less than $3.8 \%$ of the data were missing for all the variables used in the analysis. Complete case analysis was conducted, and Taylor linearization methods were used in all analyses to account for the sample weighting and complex study design. Results from the logistic regression analyses are presented as odds ratios with $95 \%$ confidence intervals (Cls), and the statistical significance level was set at $p<0.05$, a priori.

\section{Results}


The final sample included 127,097 adolescents aged 13-17 years (mean age $=14.6$ years, standard deviation $=1.2 ; 52 \%$ male). The sample of each country ranged from 838 (Liberia) to 24274 (Malaysia) as provided in Table 1. Overall, the prevalence of AST and suicide attempts was 37.0\% (5 or more days for walking or biking) and $11.6 \%$ (having at least one suicide attempt), respectively. The prevalence rates varied greatly across the included countries: AST: $12.8 \%$ (Timor-Leste) to $68.5 \%$ (Benin), suicide attempts: 3.7\% (Indonesia) to $61.0 \%$ (Samoa) (Table 1).

Figure 1 shows the prevalence of suicide attempts by being active and passive in school travel, respectively. Specifically, in the samples with AST, the prevalence of having at least 1 suicide attempt was $9.2 \%$, which was lower than passive in school travel counterparts $(12.9 \%)$.

Table 2 details the association between AST and suicide attempts in the samples. In the fully adjusted model, adolescents with AST were less likely to attempt suicide $(\mathrm{OR}=0.82,95 \% \mathrm{Cl}: 0.75-0.90, p<0.001)$. Among boys, those who had AST also had a lower odds ratio for suicide attempts $(\mathrm{OR}=0.76,95 \% \mathrm{Cl}$ : $0.64-0.90, p<0.005)$. A similarly significant relationship was also observed in girls $(\mathrm{OR}=0.87,95 \% \mathrm{Cl}$ : $0.78-0.98, p<0.05)$. Country-wise analysis showed that AST was significantly and negatively associated with suicide attempts in all countries (Figure 2). The pooled OR $(95 \% \mathrm{Cl})$, obtained by meta-analysis with random effects, was $0.86(0.79-0.92)$ with large between-country inconsistencies. Of all the 34 included countries, the significant relationship between AST and suicide attempts was observed in 8 countries (Mongolia, Philippines, Vanuatu, Honduras, Ghana, Suriname, Nepal, and Lao People's Democratic Republic).

\section{Discussion}

\subsection{General findings}

In relation to the putative role of PA (including AST) in mood regulation, to the best of our knowledge, no previous study has ever directly explored the association between AST and suicide attempts among school-based children and adolescents. Therefore, given the paucity of evidence in this domain, we sought to delineate the relationship between AST and suicide attempts, by using data from 34 LMICs. The main findings were; (i) adolescents with AST had an $18 \%$ decrease in odds for suicide attempts, compared to those without AST among more than 127,000 adolescents aged 13-17 years from 34 LMICs; (ii) there was a large level of between-country heterogeneity in the association between AST and suicide attempts; and (iii) the association between AST and attempt suicide may not vary by gender.

\subsection{Interpretations of the findings}

This is the first study examining the association between AST and suicide attempts among LMIC adolescents aged between 13 and 17 years old. Owing to the novelty of this study on the topic (AST and suicide attempts), there was no sufficient evidence or studies for direct comparison with our study. Indeed, there are no studies available to confirm or refute the research findings in the current study; Therefore, there is an urgent need for more empirical research, to further discern the relationship between 
AST and suicide attempts among adolescents. Despite little supportive and comparable evidence, some plausible explanations for promising results can be posited in the given context. First, AST has been shown to negatively associate with depressive symptoms among adolescents $[18,19]$, and there is evidence to suggest that the presence of depressive symptoms is an important predictor of attempted suicide. Collectively, AST may result in exercise-induced physiological changes [31,32] that are associated with improved emotion regulation ability that could alleviate depressive symptom, which in turn makes the odds for suicide attempts lower $[9,33,34]$. Second, as a unique tenet of PA, AST is a multifaceted behavior interacting with social and environmental settings. Social and environmental interactions during school travel, like talking with friends, are associated with better subjective well-being [35] (e.g., positive mood) [20], which, in turn, would likely reduce the likelihood of suicide attempts [3, 21]. However, these effects are assumed and more empirical evidence is needed to confirm the veracity of such claims. Another possible explanation involves bullying and victimization among adolescents. Indeed, LMIC adolescents have a greater likelihood of being bullied and victimized, compared with those living in economically advantaged countries [36]. Of note, some individual studies have found that adolescents with AST might be associated with lower odds of bully-victim experience [36, 37]. Moreover, it has been shown that bullying and victimization can cause suicide attempts, where suffering from more bullying and victimization is a contributor to suicide attempts [23]. Collectively, engaging in AST may reduce the risks of being bullied and victimized that may occur in school buses or other means of passive transportation, which may subsequently decrease the risks of suicidal attempts in adolescents. However, the underlying mechanism linking AST and suicide attempts warrants further investigation. In the present study, we only focused on the social and environmental interpretations for the association between AST and suicide attempts. Future studies are strongly encouraged in order to clarify the mechanistic association between AST and suicide attempts among adolescents.

Unlike the association between overall PA and suicide attempts [7] among adolescents (boys: negatively correlated; girls: positively correlated), the present study indicated that AST was negatively associated with suicide attempts in both boys and girls. Despite the unclear mechanisms linking overall PA and suicide attempts in girls, body dissatisfaction may be a contributory factor [38]. For instance, adolescents who demonstrated low PA level have been shown to associate with body image dissatisfaction that is predictive of suicidal ideation $[39,40]$. However, there is no evidence to show that AST has a negative role in causing other mental disorders that are positively correlated with suicide attempts. In this regard, AST could be used as a protective approach to reduce suicide attempts irrespective of gender. Notably, the mechanism linking AST and suicide attempts, and the role of gender with respect to the mechanism should be clarified in future research. Addressing this interesting research gap would be beneficial to develop more effective prevention for suicide attempts.

The present study also found that there was large inconsistency in the association between AST and suicide attempts across the included countries $(R=63 \%, p<0.01)$, indicating the studied association may vary greatly, owing to differences in social, psychological, cultural, and environmental attributes in those samples. There are some hypotheses to explain the discrepant associations. The substantial 
differences may be related to what individuals see and experience during their travel to and from school. For instance, it has been shown that events that occur during travel are also significantly related with mood [35]. This implies that only having AST is insufficient to explain mood and any resultant mental disorders. Another putative reason for the observed difference may be that the correlates or determinants of suicide attempts in different populations are different and idiosyncratic. This would reduce the relative importance of AST in explaining suicide attempts. It is also possible that the substantial difference in the association across the countries may be related to respective mental health policies. Indeed, owing to multiple countries' socioeconomic conditions, available resources, healthcare leadership and political situations, their investments in mental health policies and programs may vary considerably. If some countries lacked mental health policies and services, adolescent in those countries are likely to have poorer mental health and well-being, which in turn, may inflate the prevalence of suicide attempts. Despite these hypotheses, more detailed explanations are required to explicate the between-country differences found in this study, with greater consideration given to specific countries with regards to suicide attempts and AST among adolescents.

\subsection{Policy implications of this study}

AST among adolescents has long been beneficially associated with numerous physical and mental health outcomes [16-19], and our study provides further insights into the potential prevention of suicide attempts resultant from poorer mental health outcomes. In LMICs, there is an informative need for promotion and encouragement of AST because of its accessibility and availability. This may require the integration of multiple sectors (e.g., health care and urban design) to design and implement policy and action plans for AST. Additionally, future research should focus on the aforementioned mechanisms linking AST with suicide attempts. This may involve investigating the biological effects of AST on health, but also the context of AST (e.g., where and how). Finally, further research should be conducted to examine whether interventions that increase AST, are also effective in reducing suicidal behavior.

\subsection{Study limitations and strengths}

The use of nationally representative data from 34 countries, from different continents, and the large sample size are major strengths of this study. In addition, to the best of the authors' knowledge, this study is the first one to investigate the association between AST and suicide attempts in LMICs, which provides clinical relevance for public health and education sectors. Nonetheless, this study has several limitations that should be considered. First, there were no data available on the type of AST, and such data may have led to a better understanding of the association between AST and suicide attempts. Second, all variables were self-reported, and therefore, the subjective nature of the assessments make them susceptible to recall and reporting biases. Third, there was a difference in the timeframe between AST (past 7 days) and suicide attempts (past 12 months). That being said, it is unlikely that AST largely changes within a timeframe of 12 months. Fourth, this study included adolescents attending school, and therefore our findings may not be generalizable to those not attending school. Finally, since this was a cross-sectional study, no causal or temporal inferences can be made. 


\section{Conclusion}

This study presents previously unreported, multi-national, evidence of the association between AST and suicide attempts among adolescents from LMICs, indicating that AST may be an effective avenue for reducing suicide attempts. Future longitudinal research is required to elucidate the directionality of the variables in our study and outline potential mechanisms and explore culturally specific variations.

However, AST, could represent an approach to promote better mental health and protect against suicide attempts. The findings of our study can also help to inform suicidal behavior prevention, but should be operationalized with caution when implementing AST for reduced suicide attempts in different LMIC countries.

\section{Abbreviations}

AST: Active school travel; LMICs: Low- and Middle-income countries; PA: Physical activity; WHO: World Health Organization; GSHS: Global School-based Student Health Survey; Cis: Confidence intervals; NIHR: National Institute for Health Research.

\section{Declarations}

\section{Ethics approval and consent to participate}

Not applicable.

\section{Not applicable}

Not applicable.

\section{Availability of data and materials}

Not applicable.

\section{Competing interests}

The authors declare that they have no competing interests.

\section{Funding}

This work was supported by the Health Education England and the National Institute for Health Research (NIHR) [grant numbers ICA-CL-2017-03-001]; the NIHR Biomedical Research Centre at South London and Maudsley NHS Foundation Trust; the Medical Research Council and Guys and St Thomas Charity; and ASICS Europe BV.

\section{Authors' contributions}


STC and LYZ conceived and designed the study; STC, QY, BS, CC, ZHZ, MYZ analyzed data and interpreted the findings; $A Y, M G G, L Y Z$ proofed reading the article; $B S, M M H, A Y, M D G$ provided the language help; STC and LYZ drafted this manuscript. All authors were involved in writing the paper, had final approval of the submitted and published version, and agree with authorship.

\section{Acknowledgements}

Not applicable.

\section{References}

1. Organization WH. Suicide in the world: Global Health Estimates. 2019.

2. Bostwick JM, Pabbati C, Geske JR, McKean AJ. Suicide attempt as a risk factor for completed suicide: even more lethal than we knew. American journal of psychiatry. 2016; 173(11):1094-1100.

3. Organization WH. Preventing suicide: A global imperative. 2019.

4. Jacob L, Stubbs B, Firth J, Smith L, Haro JM, Koyanagi A. Fast food consumption and suicide attempts among adolescents aged 12-15 years from 32 countries. Journal of Affective Disorders. 2020; 266:63-70.

5. Smith L, Jackson SE, Vancampfort D, Jacob L, Firth J, Grabovac I, McDermott D, Yang L, LopezSanchez GF, Niederkrotenthaler T. Sexual behavior and suicide attempts among adolescents aged 12-15 years from 38 countries: A global perspective. Psychiatry Research. 2020; 287:112564.

6. Jacob L, Stubbs B, Koyanagi A. Consumption of carbonated soft drinks and suicide attempts among 105,061 adolescents aged 12-15 years from 6 high-income, 22 middle-income, and 4 low-income countries. Clinical Nutrition. 2020; 39(3):886-892.

7. Felez-Nobrega M, Haro JM, Vancampfort D, Koyanagi A. Sex difference in the association between physical activity and suicide attempts among adolescents from 48 countries: A global perspective. Journal of Affective Disorders. 2020; 266:311-318.

8. Vancampfort D, Stubbs B, Mugisha J, Firth J, Van Damme T, Smith L, Koyanagi A. Leisure-time sedentary behavior and suicide attempt among 126,392 adolescents in 43 countries. Journal of affective disorders. 2019; 250:346-353.

9. Vancampfort D, Hallgren M, Firth J, Rosenbaum S, Schuch FB, Mugisha J, Probst M, Van Damme T, Carvalho AF, Stubbs B. Physical activity and suicidal ideation: A systematic review and metaanalysis. J Affect Disord. 2018; 225:438-448.

10. Sibold J, Edwards E, Murray-Close D, Hudziak JJ. Physical Activity, Sadness, and Suicidality in Bullied US Adolescents. Journal of the American Academy of Child \& Adolescent Psychiatry. 2015; 54(10):808-815.

11. Jodi LS, Shimin Z, Mark D, Yan C, Deborah Leachman S. Relationship Between Physical Activity and Suicidal Behaviors Among 65,182 Middle School Students. Journal of Physical Activity and Health. 2016; 13(8):809-815. 
12. Tao FB, Xu ML, Kim SD, Sun Y, Su PY, Huang K. Physical activity might not be the protective factor for health risk behaviours and psychopathological symptoms in adolescents. Journal of paediatrics and child health. 2007; 43(11):762-767.

13. Lee C G, Cho Y, Yoo S. The relations of suicidal ideation and attempts with physical activity among Korean adolescents[J]. Journal of physical activity and health. 2013; 10(5): 716-726.

14. Tremblay MS, Barnes JD, Gonzalez SA, Katzmarzyk PT, Onywera VO, Reilly JJ, Tomkinson GR, Global Matrix 20 Res T. Global Matrix 2.0: Report Card Grades on the Physical Activity of Children and Youth Comparing 38 Countries. Journal of Physical Activity \& Health. 2016; 13(11):S343-S366.

15. Aubert S, Barnes JD, Abdeta C, Nader PA, Adeniyi AF, Aguilar-Farias N, Andrade Tenesaca DS, Bhawra J, Brazo-Sayavera J, Cardon G et al. Global Matrix 3.0 Physical Activity Report Card Grades for Children and Youth: Results and Analysis From 49 Countries. Journal of Physical Activity \& Health. 2018; 15:S251-S273.

16. Lubans DR, Boreham CA, Kelly P, Foster CE. The relationship between active travel to school and health-related fitness in children and adolescents: a systematic review. International Journal of Behavioral Nutrition and Physical Activity. 2011; 8(1):5.

17. Schoeppe S, Duncan MJ, Badland H, Oliver M, Curtis C. Associations of children's independent mobility and active travel with physical activity, sedentary behaviour and weight status: A systematic review. Journal of Science and Medicine in Sport. 2013; 16(4):312-319.

18. Sun Y, Liu Y, Tao FB. Associations Between Active Commuting to School, Body Fat, and Mental Wellbeing: Population-Based, Cross-Sectional Study in China. Journal of Adolescent Health. 2015; 57(6):679-685.

19. Gu J, Chen S-T. Association between Active Travel to School and Depressive Symptoms among Early Adolescents. Children. 2020; 7(5):41.

20. Waygood EOD, Friman M, Olsson LE, Taniguchi A. Transport and child well-being: An integrative review. Travel Behaviour \& Society. 2017; 9:32-49.

21. Klonsky ED, May AM, Saffer BY. Suicide, Suicide Attempts, and Suicidal Ideation. Annual Review of Clinical Psychology. 2016; 12(1):307-330.

22. Pengpid S, Peltzer K. Prevalence and correlates of past 12-month suicide attempt among in-school adolescents in Guatemala. Psychology research and behavior management. 2019; 12:523.

23. Koyanagi A, Oh H, Carvalho AF, Smith L, Haro JM, Vancampfort D, Stubbs B, DeVylder JE. Bullying Victimization and Suicide Attempt Among Adolescents Aged 12-15 Years From 48 Countries. Journal of the American Academy of Child and Adolescent Psychiatry. 2019; 58(9):907-+.

24. Liu X, Huang Y, Liu Y. Prevalence, distribution, and associated factors of suicide attempts in young adolescents: School-based data from 40 low-income and middle-income countries. Plos One. 2018; 13(12).

25. Sharma B, Nam EW, Kim HY, Kim JK. Factors associated with suicidal ideation and suicide attempt among school-going urban adolescents in Peru. International journal of environmental research and public health. 2015; 12(11):14842-14856. 
26. Núñez-González S, Lara-Vinueza A, Gault C, Delgado-Ron A. Trends and Spatial Patterns of Suicide Among Adolescent in Ecuador, 1997-2016. Clinical Practice \& Epidemiology in Mental Health. 2018; 14:283-292.

27. Felez-Nobrega M, Haro JM, Vancampfort D, Koyanagi A. Sex difference in the association between physical activity and suicide attempts among adolescents from 48 countries: A global perspective. Journal of Affective Disorders. 2020; 266:311-318.

28. Prochaska JJ, Sallis JF, Long B. A physical activity screening measure for use with adolescents in primary care. Archives of pediatrics \& adolescent medicine. 2001; 155(5):554-559.

29. Vancampfort D, Stubbs B, Firth J, Van Damme T, Koyanagi A. Sedentary behavior and depressive symptoms among 67,077 adolescents aged 12-15 years from 30 low- and middle-income countries. International Journal of Behavioral Nutrition and Physical Activity. 2018; 15(1):73.

30. Higgins JP, Thompson SG. Quantifying heterogeneity in a meta-analysis. Statistical Medicine. 2002; 21.

31. Doré I, Sylvester B, Sabiston C, Sylvestre M-P, O'Loughlin J, Brunet J, Bélanger M. Mechanisms underpinning the association between physical activity and mental health in adolescence: a 6-year study. International Journal of Behavioral Nutrition and Physical Activity. 2020;17(1):9.

32. Bell SL, Audrey S, Gunnell D, Cooper A, Campbell R. The relationship between physical activity, mental wellbeing and symptoms of mental health disorder in adolescents: a cohort study. International Journal of Behavioral Nutrition and Physical Activity. 2019; 16(1):138.

33. Andermo S, Hallgren M, Nguyen TT, Jonsson S, Petersen S, Friberg M, Romqvist A, Stubbs B, Elinder LS. School-related physical activity interventions and mental health among children: a systematic review and meta-analysis. Sports Med Open. 2020; 6(1):25.

34. Lin K, Stubbs B, Zou W, Zheng W, Lu W, Gao Y, Chen K, Wang S, Liu J, Huang Y et al. Aerobic exercise impacts the anterior cingulate cortex in adolescents with subthreshold mood syndromes: a randomized controlled trial study. Transl Psychiatry. 2020; 10(1):155.

35. Ramanathan S, O'Brien C, Faulkner G, Stone M. Happiness in motion: emotions, well-being, and active school travel. J Sch Health. 2014; 84(8):516-523.

36. Alfonso-Rosa RM, García-Hermoso A, Sanders T, Parker P, Oriol-Granado X, Arnott H, del Pozo Cruz B. Lifestyle behaviors predict adolescents bullying victimization in low and middle-income countries. Journal of Affective Disorders. 2020; 273:364-374.

37. Sampasa-Kanyinga H, Chaput J-P, Hamilton HA, Larouche R. School bus travel is associated with bullying victimization among Canadian male, but not female, middle and high school students. Child Abuse \& Neglect. 2016; 58:141-148.

38. Lawler M, Nixon E. Body dissatisfaction among adolescent boys and girls: the effects of body mass, peer appearance culture and internalization of appearance ideals. Journal of youth and adolescence. 2011; 40(1):59-71.

39. Kim D-S. Body image dissatisfaction as an important contributor to suicidal ideation in Korean adolescents: gender difference and mediation of parent and peer relationships. Journal of 
psychosomatic Research; 2009. 66(4):297-303.

40. Kim D-S, Kim H-S. Body-image dissatisfaction as a predictor of suicidal ideation among Korean boys and girls in different stages of adolescence: A two-year longitudinal study. Journal of Adolescent Health. 2009; 45(1):47-54.

\section{Tables}

Table 1. Sample characteristics by each country included in this study 


\begin{tabular}{|c|c|c|c|c|}
\hline Country & Year & $\mathrm{n}$ & Active school travel a & Suicide attempt ${ }^{b}$ \\
\hline Overall & I & 127097 & $37.0 \%$ & $11.6 \%$ \\
\hline Argentina & 2014 & 23063 & $47.0 \%$ & $16.4 \%$ \\
\hline Bangladesh & 2014 & 2552 & $53.1 \%$ & $6.8 \%$ \\
\hline Belize & 2011 & 1399 & $42.2 \%$ & $14.4 \%$ \\
\hline Benin & 2016 & 1311 & $68.5 \%$ & $13.2 \%$ \\
\hline Bolivia & 2012 & 2905 & $41.6 \%$ & $20.8 \%$ \\
\hline Cambodia & 2013 & 2660 & $52.7 \%$ & $7.0 \%$ \\
\hline Dominican Republic & 2016 & 1064 & $42.2 \%$ & $16.6 \%$ \\
\hline El Salvador & 2013 & 1620 & $42.9 \%$ & $13.3 \%$ \\
\hline Fiji & 2016 & 2582 & $27.7 \%$ & $11.1 \%$ \\
\hline Ghana Junior High & 2012 & 1086 & $29.3 \%$ & $28.7 \%$ \\
\hline Guatemala & 2015 & 3301 & $33.3 \%$ & $15.9 \%$ \\
\hline Honduras & 2012 & 1316 & $41.1 \%$ & $17.5 \%$ \\
\hline Indonesia & 2015 & 2877 & $36.6 \%$ & $3.7 \%$ \\
\hline Kiribati & 2011 & 1370 & $33.1 \%$ & $31.5 \%$ \\
\hline Lao People's Democratic Republic & 2015 & 3402 & $30.3 \%$ & $5.1 \%$ \\
\hline Lebanon & 2017 & 3948 & $14.8 \%$ & $9.9 \%$ \\
\hline Liberia & 2017 & 838 & $23.7 \%$ & $29.3 \%$ \\
\hline Malaysia & 2012 & 24274 & $33.0 \%$ & $6.7 \%$ \\
\hline Mauritius & 2017 & 2575 & $28.8 \%$ & $12.8 \%$ \\
\hline Mongolia & 2013 & 4233 & $63.7 \%$ & $9.6 \%$ \\
\hline Mozambique & 2015 & 996 & $18.3 \%$ & $16.9 \%$ \\
\hline Namibia & 2013 & 2779 & $31.1 \%$ & $25.9 \%$ \\
\hline Nepal & 2015 & 5166 & $40.6 \%$ & $10.1 \%$ \\
\hline Peru & 2010 & 2559 & $47.3 \%$ & $17.2 \%$ \\
\hline Philippines & 2015 & 7367 & $29.7 \%$ & $16.8 \%$ \\
\hline Samoa & 2011 & 1370 & $23.9 \%$ & $61.0 \%$ \\
\hline Solomon Islands & 2011 & 977 & $27.8 \%$ & $35.5 \%$ \\
\hline
\end{tabular}




\begin{tabular}{|lllll|} 
Sri Lanka & 2016 & 2934 & $41.6 \%$ & $6.8 \%$ \\
\hline Suriname & 2016 & 1520 & $32.2 \%$ & $11.4 \%$ \\
\hline Thailand & 2015 & 4375 & $26.9 \%$ & $13.3 \%$ \\
\hline Timor-Leste & 2015 & 2289 & $12.8 \%$ & $9.6 \%$ \\
\hline Tonga & 2017 & 2126 & $28.7 \%$ & $15.2 \%$ \\
\hline United Republic of Tanzania & 2014 & 2559 & $24.6 \%$ & $10.4 \%$ \\
\hline Vanuatu & 2016 & 1704 & $31.2 \%$ & $22.5 \%$ \\
\hline
\end{tabular}

a having walked or cycled to school at least 5 days per week.

${ }^{b}$ having at least one suicide attempt during the past 12 months.

Table 2. Association between active school travel and suicide attempts over the past 12 months estimated by multivariable logistic regression (by overall and gender)

\begin{tabular}{|c|c|c|c|c|c|c|c|c|c|}
\hline & \multicolumn{3}{|c|}{ Overall ${ }^{a}$} & \multicolumn{3}{|l|}{ Boys ${ }^{b}$} & \multicolumn{3}{|c|}{ Girls b } \\
\hline & OR & $95 \% \mathrm{C}$ & & OR & $95 \%$ & & OR & $95 \% \mathrm{C}$ & \\
\hline Passive & \multicolumn{3}{|c|}{ Reference group } & \multicolumn{3}{|c|}{ Reference group } & \multicolumn{3}{|c|}{ Reference group } \\
\hline Active $^{c}$ & $0.82^{\star \star \star}$ & 0.75 & 0.90 & $0.76^{* *}$ & 0.64 & 0.90 & $0.87^{\star}$ & 0.78 & 0.98 \\
\hline
\end{tabular}

a denotes controlling for country, gender, age, food insecurity, presence of close friends, anxiety-induced insomnia, fruit and vegetable intake, fast food consumption, alcohol consumption, physical activity and sedentary behavior; ${ }^{\text {b }}$ denotes controlling for country, age, food insecurity, presence of close friends, anxiety-induced insomnia, fruit and vegetable intake, fast food consumption, alcohol consumption, physical activity and sedentary behavior; ${ }^{c}$ denotes reporting at least 5 days of active school travel per week; ${ }^{* \star} p<0.05, * \star p<0.005$, *** $p<0.001$.

\section{Figures}




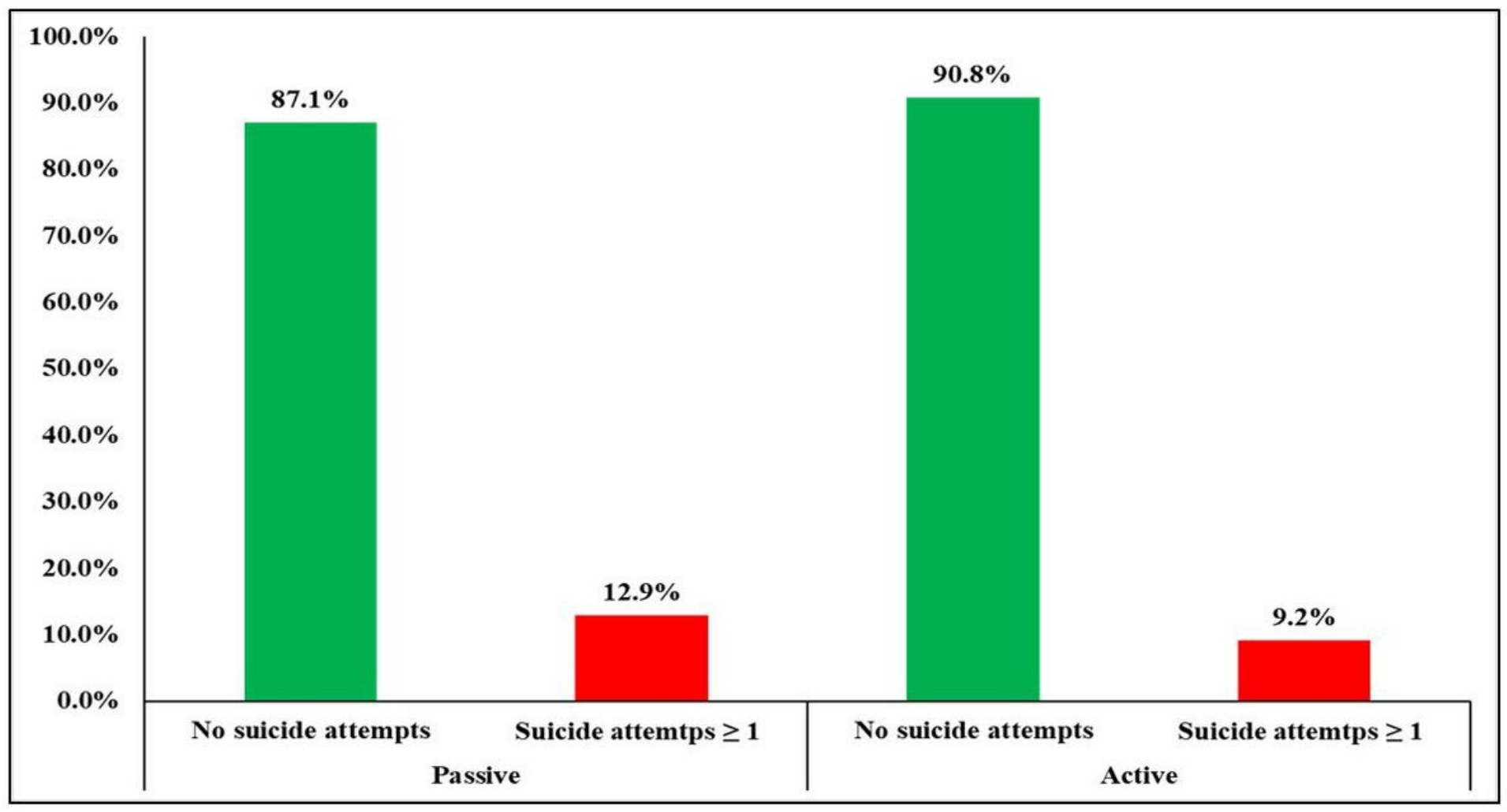

\section{Figure 1}

Prevalence of suicide attempt by being active and passive in school travel. 


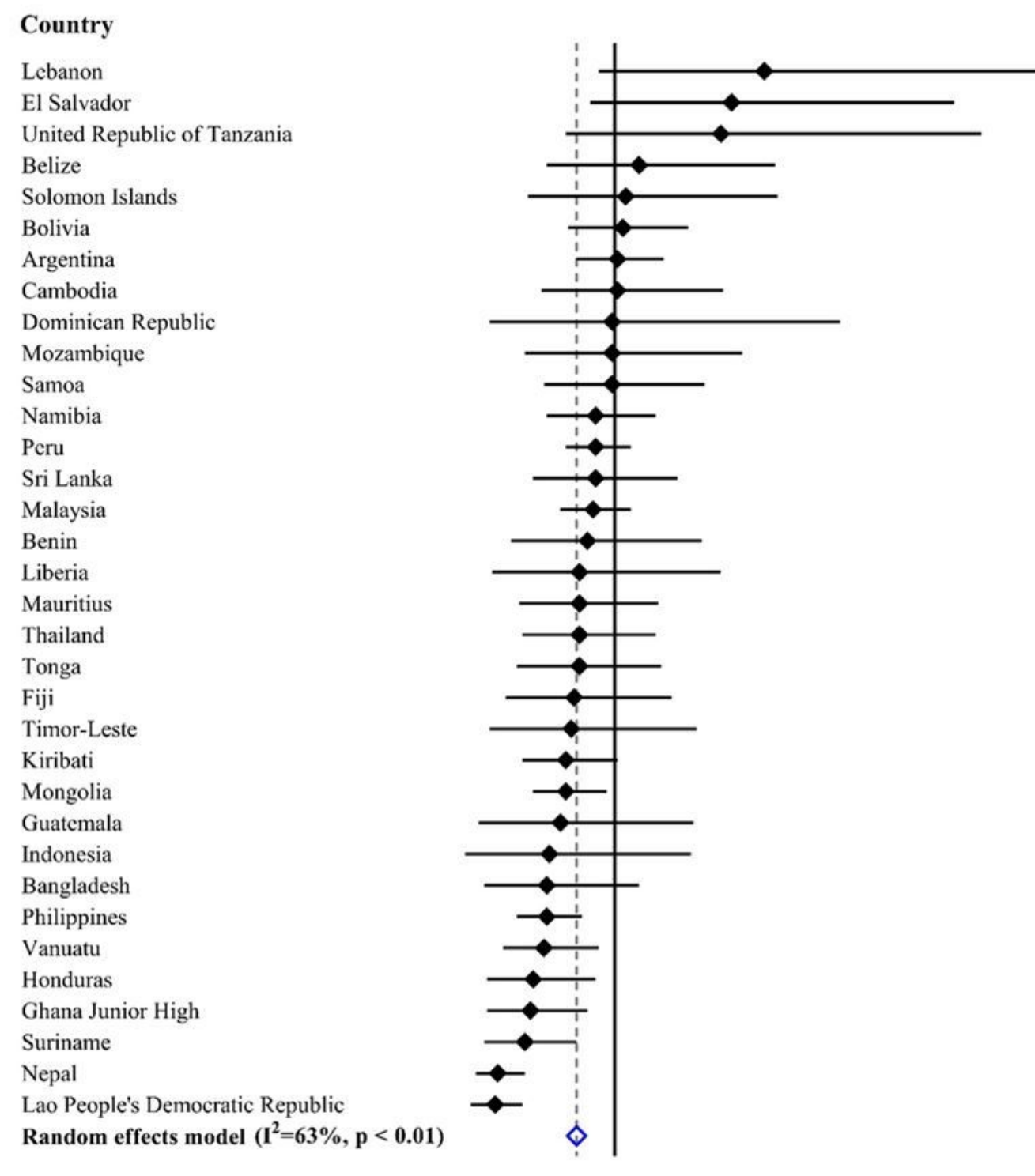

OR $(95 \% \mathrm{CI})$

$1.55(0.94,2.57) \quad 1.6$

$1.43 \quad(0.91,2.25) \quad 1.8$

$1.39 \quad(0.82,2.35) \quad 1.5$

$1.09(0.75,1.59) \quad 2.3$

$1.04(0.68,1.60) \quad 2.0$

$1.03(0.83,1.27) \quad 3.8$

$1.01 \quad(0.86,1.18) \quad 4.4$

$\begin{array}{lll}1.01 & (0.73,1.40) & 2.7\end{array}$

$0.99(0.54,1.83) \quad 1.2$

$0.99(0.67,1.47) \quad 2.2$

$0.99(0.74,1.33) \quad 3.0$

$\begin{array}{lll}0.93 & (0.75,1.15) & 3.8\end{array}$

$\begin{array}{lll}0.93 & (0.82,1.06) & 4.7\end{array}$

$0.93(0.70,1.23) \quad 3.1$

$0.92(0.80,1.06) \quad 4.6$

$0.90 \quad(0.62,1.32) \quad 2.3$

$0.87(0.55,1.39) \quad 1.8$

$0.87(0.65,1.16) \quad 3.0$

$0.87(0.66,1.15) \quad 3.2$

$0.87(0.64,1.17) \quad 2.9$

$0.85(0.60,1.21) \quad 2.5$

$0.84 \quad(0.54,1.30) \quad 1.9$

$0.82(0.66,1.01) \quad 3.8$

$\begin{array}{lll}0.82 & (0.70,0.97) & 4.3\end{array}$

$\begin{array}{lll}0.80 & (0.50,1.29) & 1.7\end{array}$

$0.76(0.45,1.28) \quad 1.5$

$0.75 \quad(0.52,1.09) \quad 2.3$

$\begin{array}{lll}0.75 & (0.64,0.88) & 4.4\end{array}$

$0.74(0.59,0.94) \quad 3.6$

$0.70(0.53,0.93) \quad 3.1$

$\begin{array}{lll}0.69 & (0.53,0.90) & 3.2\end{array}$

$0.67(0.52,0.86) \quad 3.4$

$0.57(0.49,0.67) \quad 4.4$

$0.56(0.47 .0 .66) \quad 4.3$

$0.86(0.79,0.92) \quad 100.0$

\section{Figure 2}

Country-wise associations between active school travel and suicide attempts estimated by multivariable logistic regression. Abbreviation: OR Odds ratio; $\mathrm{Cl}$ Confidence interval. The pooled estimate was obtained by meta-analysis with random effects model. 